\title{
LA EDUCACIÓN FÍSICA EN POLÍTICAS SOCIO-EDUCATIVAS DESTINADAS A LOS JÓVENES*
}

\author{
LIC. ALEJO LEVORATTI \\ Universidad Nacional de La Plata/Universidad Nacional de Quilmes (Buenos Aires - Argentina) \\ E-mail: levoratti@gmail.com
}

\begin{abstract}
RESUMEN
Este trabajo tiene como propósito abordar las problemáticas de las prácticas corporales inmersas en las políticas socio-educativas de la Dirección General de Cultura y Educación (DGCyE) de la Provincia de Buenos Aires Argentina y, en particular, el sentido y la función asignada a ellas en el programa "Patios Abiertos en las Escuelas" programa destinado a los jóvenes. Manteniendo en dicho abordaje comparaciones con otras investigaciones y con documentos de iniciativas similares que se desarrollan en Brasil: "Abriendo Espaços" ejecutado por la Organización de las Naciones Unidas para la Educación, la Ciencia y la Cultura (Unesco) y el "Programa Estadual de Esporte Escolar" del estado de Santa Catarina y el programa "Segundo Tempo" es una iniciativa del Ministerio do Esporte, indagando sobre las líneas de fundamentación asignadas.
\end{abstract}

PALABRAS CLAVES: Educación fisica; politicas socio-educativas; jovenes; prácticas corporales.

* El presente trabajo no conto con apoyo financiero de ninguna naturaleza para su realización. Manifiesto que no hubo conflictos de intereses para la realización del presente estudio. 


\section{INTRODUCCIÓN}

Este trabajo aborda la problemática de las prácticas corporales inmersas en programas que se encuentran dentro de las políticas sociales que se desarrollan en materia educativa en la Argentina, debiendo aclarar que este tipo de experiencias, orientadas a los jóvenes se vienen reproduciendo tanto en el país como en el extranjero, focalizando el análisis en esta oportunidad en el sentido y en la función asignada a las prácticas corporales que se desarrollan en el programa "Patios Abiertos en las Escuelas" a partir del análisis de los documentos centrales de esta propuesta. Esta iniciativa es desarrollada por la Dirección General de Cultura y Educación de la Provincia de Buenos Aires, en Argentina, y se encuentra destina a niños y jóvenes, de II a 2 I años de edad, en situación de "vulnerabilidad social"'.

Aunque las prácticas corporales son una de las tantas categorías temáticas de los proyectos, éstas tienen un lugar central dentro de la experiencia, por un lado por la cantidad de escuelas en donde se llevan a cabo, por otro por el impacto en la población destinataria².

Para poder realizar un abordaje de esta problemática, se procedió a efectuar una contextualización sobre la situación juvenil, dado que la misma nos permite construir un contexto en donde analizar estas experiencias y la inclusión de las prácticas corporales.

Entrando en la propuesta del programa, se analizaron en primera instancia los actos administrativos de esta iniciativa, centrándose en las fundamentaciones que se le asignan a la inclusión disciplinar, con el fin de abrir el espectro y mostrar la amplitud de esta problemática. Asimismo se presentarán brevemente distintas experiencias con caracteres similares en funcionamiento en el Brasil.

\section{ACLARACIONES METODOLÓGICAS}

Las fuentes de esta investigación serán de carácter bibliográfico/documental dado que la información con la se trabajará se encuentra en mayor medida en documentos, resoluciones, proyectos en ejecución, no sistematizados y que no han recibido hasta el momento un tratamiento analítico. Otra fuente importante a

I. Es pertinente aclarar que las edades de los participantes ha sufrido modificaciones a lo largo de la implementación del programa, los datos presentados corresponden al Manual de Procedimientos del año 2006, en la actualidad la franja poblacional se extiende desde los 5 a los 21 años.

2. Categorías Temáticas de los proyectos del programa "Patios Abiertos en las Escuelas" pueden ser: arte y cultura, actividades de contención social, comunicación, participación en la comunidad, actividades de capacitación en oficios múltiples o en oficios no convencionales, prácticas corporales. 
incorporar es la bibliografía (libros y artículos en revistas especializadas), que muestran experiencias de iguales características, permitiendo dicha labor un diálogo con otros investigadores que hayan abordado esta temática (GIL, | 99|).

A modo de organizar el análisis bibliográfico/documental se procedió en primero lugar a sistematizar la información agrupándola a partir de sus caracteres intrínsecos en cuatro tipos: en documentos/fundamentaciones oficiales de los programa "Patios Abiertos en las Escuelas", estudios sobre programas sociales que incluyan las prácticas corporales, informes sobre la situación de la población destinataria, estudios sobre la incorporación de las prácticas corporales en distintos tipos de programas, para luego realizar un trabajo sistemático sobre la misma.

\section{LOS JÓVENES VISTOS COMO RIESGO POTENCIAL}

La Juventud y la Vejez no están dadas, sino que se construyen socialmente en la lucha entre jóvenes y viejos

(Piere Bourdieu, 1990, p. 164)

La intención de este apartado es presentar una problematización sobres los jóvenes, y en particular de éstos como destinatarios de políticas sociales. Con tal fin se trabajará sobre las representaciones sociales que se tienen de estos, y la construcción de esta población como destinataria de políticas sociales focalizadas, a partir de fuentes bibliográficas.

En las últimas décadas, la preocupación sobre las poblaciones juveniles, se encuentra siempre en primera plana, con una fuerte asociación de los jóvenes con los problemas de inseguridad, violencia, delincuencia, homicidios. Esta serie de discursos reforzados, naturalizados y agudizados por la prensa, los transforman en los enemigos internos y chivos expiatorios de la sociedad, como destaca Rosana Reguillo (1997). En las representaciones sociales de los jóvenes, en la Provincia de Buenos Aires - Argentina -, que presenta Mariana Chaves (2005), realizadas a partir de la utilización de fuentes primarias y secundarias, se observa a éstos como: inseguros de sí mismos, en etapa de transición, no productivos, incompletos, desinteresados o sin deseo, desviados o con posibilidad de desviarse, ser peligrosos, victimados, rebeldes o revolucionarios, o ser del futuro. Se visualiza, como destaca la autora, en todas estas afirmaciones una visión negativa o negativizada en relación a los jóvenes; pero a su vez podemos ver como todas las construcciones que se realizan, son a partir de una visión adulta, en la cual éste es considerado inferior por no acercarse al estado de completud del adulto construido por el propio "adulto" de los sectores más influyentes de la sociedad. 
Este tipo de figuraciones no son exclusivas de la actualidad, por el contrario se presentaron en distintos momentos de la historia del occidente, focalizándose la negativización sobre distintos aspectos, los que dependían del período y de los problemas sociales imperantes. En todos los casos, se mantuvo una carga de señalización, perdiendo la referencia de los jóvenes o de las culturas juveniles, trasformándose esas personificaciones en una serie de consumos, los cuales los colocan de un lado o de otro: ser consumidor de productos que los definen como jóvenes, o ser infamados como peligrosos, limitando su accionar a través de la participación en políticas sociales para disminuir los riegos al resto de la sociedad.

Si las juventudes o las culturas juveniles no se pueden definir o caracterizar con una categoría generacional, que se encuentra de paso para la adultez, icuál es el carácter por donde se centra la estigmatización? ¿Qué culturas juveniles son peligrosas? o ise debe a cuestiones de acumulación de determinados capitales, a una determinada capacidad de consumo, a una determinada relación con la tecnología y las instituciones? Si esta categoría como destacan Reguillo y Chaves, son el chivo expiatorio de los problemas de la sociedad y del modelo económico, proceso que se legitima a partir de la negativización y negación a las cuales son sometidas las culturas juveniles, se centran en determinadas poblaciones la que varía, no por su edad cronológica, sino por su capital económico, cultural y social siendo en estos puntos donde se perfila la estigmatización.

Ampliando sobre este punto, encontramos que Rosana Reguillo (2000), considera al mismo momento histórico como de toma de visibilidad de la juventud, a raíz de tres procesos: los cambios en la reorganización económica, la aparición de una serie de productos culturales específicos para los jóvenes y el advenimiento como sujetos jurídicos, con leyes e instituciones especificas (REGUILLO, 2000, p. 22 a 26). En este mismo período es donde encontramos el primer antecedente de apertura de escuelas los fines de semana, para la realización de prácticas corporales en los Estados Unidos, escuelas que se encontraban bajo una corriente denominada "escola da comunidade" por Candido Gomes, en las cuales el deporte tenía como objeto combatir la creciente delincuencia Juvenil (Gomes, 200 I).

A partir de esta etapa, los jóvenes han sido caracterizados de distintas maneras como destaca Rossana Reguillo, respondiendo en cierta medida a la forma de sociabilización e institucionalización de esta población en los distintos momentos históricos.

En una época, fueron los sindicatos y los partidos políticos, las principales formas de agrupación. Los jóvenes encontraban en estos lugares la estructura y el espacio para su organización y sociabilización, manteniéndose siempre en espacios institucionalizados. Este modelo tenía como propósito la inexistencia de tiempos muertos, como destaca Castel, "[...] la Cultura, el Deporte, la salud, la cercanía 
con la naturaleza, las relaciones sanas (y no sexualizadas) entre los jóvenes, debían ocupar el tiempo no destinado al trabajo. Nada de tiempo muerto; libertad no era la anarquía ni el puro disfrute. Se debía proceder mejor que los burgueses, y trabajar en los ocios" (CASTEL, 1997, p. 345).

Este proceso de corrimiento de los jóvenes del ámbito de la escuela, de los centros de estudiantes, del trabajo, de los sindicatos, conlleva a un cambio en la forma de organización juvenil, como destaca Reguillo, tendiendo hacia un ámbito no institucionalizado, de autorganización (REGUILLo, 2000).

Si asociamos las representaciones sociales sobre las juventudes, los cambios políticos y el desplazamiento de estos jóvenes de las instituciones tradicionales como la escuela asociada al poder disciplinador a otros espacios, nos encontramos al decir de Foucault en palabras de Mariana Chaves con un cambio de paradigma desde las disciplinas hacia las políticas de seguridad, cuyo objetivo es gobernar el desorden. Destaca la autora que "Hoy al estado no le interesa disciplinar, sin embargo sí le interesa el mecanismo de seguridad. Ya no importa que los chicos se porten mal, el problema es que son peligrosos. No es la acción misma, sino la posibilidad de acción. Todo joven es sospechoso, carga por su estatus cronológico la marca del peligro: peligro para él mismo: irse por el mal camino, no cuidarse; peligro para su familia: traer problemas; peligro para los ciudadanos: molestar, agredir, ser violento; en fin, peligro para la sociedad: no produce nada, no respeta las normas" (CHAVEZ, 2005, p. 5). Ampliando sobre el paradigma de seguridad la autora destaca que "[... ] es el único que legitima la política conduciendo al mismo tiempo a la deslegitimación de la política" (ChAveZ, 2005, p. 5). Con este contexto de situación procederemos a analizar la documentación del programa "Patios Abiertos en las Escuelas".

\section{PROGRAMA PATIOS ABIERTOS EN LAS ESCUELAS}

El Programa Patios Abiertos en las Escuelas es una iniciativa que se encuentra bajo la dependencia de la Dirección Provincial de Política Socio-Educativa de la Dirección General de Cultura y Educación de la Provincia de Buenos Aires (DGCyE) de Argentina, la cual tiene como función, "coordinar la aplicación de los planes y programas sociales en relación a las ramas de educación" como se destaca en la resolución n. 100/04 de creación.

El programa, tiene como propósito central la inclusión educativa de la población comprendida entre los II a 2 I años ${ }^{3}$. No se encuentra limitada la pertenencia

3. Es pertinente aclarar que la documentación que se analizó del programa fue producida desde su creación hasta el mes de diciembre de 2006. 
a los alumnos que asisten regularmente al establecimiento, ni a los alumnos de otros establecimientos. Del programa, puede participar toda persona entre II a 2 I años esté o no escolarizada. Esta aclaración se debe a que otras iniciativas que se presentan en este trabajo, tienen como requisito para la integración al programa la escolarización obligatoria de los participantes, como es en el caso del programa Segundo Tempo, desarrollado por el Ministerio do Esporte do Brasil.

El Programa "Patios Abiertos en las Escuelas" consiste en la apertura de los edificios escolares los días sábados y/o domingos, para la realización de distintos tipos de actividades. Las temáticas de los talleres, dependen del proyecto que fue presentado y aprobado por la Coordinación del programa, para esa determinada sede. Tienen todas las escuelas de enseñanza pública sin importar su rama educativa y las 200 escuelas de gestión privada que se encuentran dentro de las escuelas focalizadas, posibilidad de presentar proyectos. Los talleres que se dictan en cada una de las escuelas dependen de la propuesta que se encuentra aprobado para funcionar en dicha institución.

\section{BREVE HISTORIA DEL PROGRAMA}

El programa comenzó a funcionar a partir del primero de agosto del año 2004, con la apertura de los primeros cincuenta proyectos (resolución 3328/04), previo a esto se elaboraron convenios, resoluciones, que dieron forma a lo que en un primer momento se llamó "Patios Abiertos", devenido en "Patios Abiertos en las Escuelas".

Con el propósito de realizar un recorrido histórico sobre el programa, tomaré como fuentes aquellos actos administrativos que hacen referencia a éste.

La primera resolución encontrada es la n. 4235 de la DGCyE, del ocho de octubre del año 2003, que es un protocolo de colaboración entre la Organización de las Naciones Unidas para la Educación, la Ciencia y la Cultura (Unesco Brasil), representada en ese caso por Jorge Werthein y la Dirección General de Cultura y Educación de la Provincia de Buenos Aires representada por el director general, profesor Mario Oporto.

En este convenio de colaboración, se manifiesta la necesidad de realizar acciones conjuntas, enumerándose diez objetivos, destacándose en primer lugar, la promoción de una "cultura de paz" entre los Jóvenes, en segundo lugar la promoción de valores, actitudes, compromiso social y comunitario para los jóvenes. Otro grupo de objetivos, en su mayoría, están asociados a la creación, apertura y al uso responsable de los espacios públicos, encontrando dentro de éstos la proyección de lugares para la realización de actividades recreativas, deportivas y 
artísticas con el propósito de integrar a los adolescentes entre sí, con la escuela y la comunidad.

Esta experiencia busca que los jóvenes que se hallan en situación de "vulnerabilidad social", como son caracterizados en dicho documento, o que no tienen la posibilidad de realizar actividades de su agrado, encuentren en la escuela ese espacio para efectuarlas, logrando de esta forma una interacción entre la escuela y la comunidad. Se presenta a los adolescentes de lugares marginales como no integrados, tanto a un grupo de pares, a la comunidad y a la escuela, proponiendo la realización de distintos tipos de actividades como un antídoto ante esta problemática.

Continuando con esta cronología, en el mes de julio de 2004 se firma la resolución 2273/04, por la cual se crea el "Programa Patios Abiertos en las Escuelas", estableciéndose en dicho acto administrativo lineamientos generales, sobre los objetivos del programa, que se presentarán a continuación, cuestiones relacionadas a la aprobación de los proyectos, y la creación de un manual de procedimientos, con el cual se ejecutarán las experiencias ${ }^{4}$.

El 22 de septiembre del mismo año es firmada la resolución 3328/04 por la cual se aprueban los primeros 50 proyectos del programa que se encontraban en funcionamiento desde el primero de agosto del mismo año.

Finaliza el año 2004, con I 4 proyectos, en todo el territorio provincial. Durante el transcurso del año 2005, se aprobaron en convocatorias sucesivas 208 proyectos, terminando dicho año con 319 iniciativas ${ }^{5}$.

En el 2006, es decretado el programa como de interés provincial por el Consejo General de Educación de la Provincia de Buenos Aires, por el acto administrativo n. 72/06.

\section{OBJETIVOS DEL PROGRAMA}

La resolución 2273/04 la que en sus artículos 2do y 3ro pauta la creación de un equipo de coordinación central encargado de establecer los objetivos, metas y cronogramas de ejecución, y la conformación de un "Manual Operativo o de procedimiento" ${ }^{\prime \prime}$. Me concentraré en este último documento, por ser el central del programa.

4. Se crea en la Dirección de Coordinación de Programas Dependiente de la Dirección Provincial de Políticas Socio-Educativas el Programa Patios Abiertos en las Escuelas" (resolución 2273/04).

5. Es de aclarar que durante el trascurso del año 2005 fueron dado de baja a 3 proyectos por la coordinación central del Programa, es por eso que la suma no asciende a 322.

6. Disponible en: <http://abc.gov.ar/lainstitucion/organismos/coordinacionprogramas/programas/ patiosabiertos/default.cfm>. 
En dicho manual, se encuentran enumerados 13 objetivos, pudiéndose agrupar en dos tipos, los que tienen como centro a los jóvenes y adolescentes y los que se focalizan en la escuela.

Analizando el primer grupo, que son la mayoría de los objetivos, se destaca como propósito de la iniciativa: "La retención y posible reincorporación de alumnos al sistema educativo formal [ ...]”. Éste es colocado en segundo lugar dentro de los objetivos, siendo el articulador de esta agrupación, los cuales presento manteniendo el orden de dicho documento.

En el primero se manifiesta "El desarrollo y la capacitación de los jóvenes a través de actividades no institucionalizadas", el tercero destaca "El mejoramiento de la relación de los adolescentes con la escuela, con los docentes y con la comunidad.." el quinto expresa "Promover el entendimiento del sentido de educarse", el sexto "Generar un sentimiento de pertenencia que permita al alumno identificarse con su Escuela y, al mismo tiempo, sentirla como algo propio, significativo y posible de ser modificado [...]" el octavo "La integración social de los adolescentes", el noveno "Adecuar los procesos de comunicación adulto - joven mediante el reconocimiento e incorporación de la pluralidad de códigos, símbolos y valores aportados por los últimos, tendiendo a la superación de la dicotomía producida entre las prácticas culturales generadas por la estructura formal de la Escuela y aquellas espontáneamente surgidas de los jóvenes", el décimo primero plantea "Estimular entre los docentes y miembros de la comunidad el desarrollo de un conocimiento reflexivo sobre el mundo de los jóvenes" y el décimo segundo establece "una fluida comunicación entre los establecimientos participantes, interconectando en red a los jóvenes de diversos ámbitos, fomentando el diálogo generacional, la cooperación y la integración en iniciativas conjuntas".

Los objetivos tienen como propósito que los jóvenes conozcan otra escuela o mejor dicho que construyan otra visión sobre ésta, rompiendo con la visión tradicional, para que encuentren en ella un espacio para permanecer a partir de la realización de las actividades de su agrado. Permitiendo como es planteado en las fundamentaciones, protegerlos de un afuera inseguro, o proteger a los de afuera de ellos, a partir de alejarlos de la calle. Para graficar podemos leer en las justificaciones de los proyectos en funcionamiento, de un taller de prácticas corporales, en el conurbano Bonaerense:

[...] nuestra escuela se encuentra abocada en tratar de obtener espacios disponibles para apartar a los jóvenes de la comunidad de las calles y la marginalidad, ofreciéndoles diversas actividades a desarrollarse en la escuela.

Encontrando en el interior de la provincia, experiencias apoyadas en principios similares, como se puede ver en este extracto: 
Ocupar el tiempo libre de los adolescentes, con actividades de su agrado, para revertir las situaciones de apatía, alejándolos de la calle y disminuyendo el riesgo social.

Evitar situaciones de vulnerabilidad social, disminuyendo la permanencia de los jóvenes en las calles.

Esta búsqueda por la permanencia de los jóvenes en las escuelas, como un lugar seguro de los peligros del "afuera", de la calle, del lugar donde viven cotidianamente, presenta a la escuela como una trinchera en donde cubrirse de los problemas o de los peligros de su vida corriente.

Esta circunstancia tiene como problema de fondo, por un lado la estigmatización de la cual son blancos los jóvenes, como se presentó anteriormente, en donde la situación de pobreza en la cual viven, agravada por la falta de espacios públicos o de posible acceso, por cuestiones económicas, los transforma en no consumidores, por lo tanto en peligrosos. Esto se produce por la privatización de los espacios públicos, como es el caso de las plazas o potreros que se trasformaron en emprendimientos privados, los clubes que se encuentran en mal estado financiero y administrados por iniciativas de carácter privado, por la imposibilidad de asistir a actividades culturales como a espectáculo deportivos, cines o la participación en encuentros deportivos porque la mayoría de los lugares son privados.

Es necesario destacar que todos los extractos presentados provienen, de aquellas experiencias que tienen como temática a las prácticas corporales, mostrándonos una de las principales fundamentaciones disciplinares dentro del programa.

La segunda serie de objetivos, que encontramos en el Manual Operativo 2006 del Programa, están asociados a la reconstrucción del vínculo de la escuela con la comunidad, siendo esta intención limitada ya que la población que puede participar es la comprendida entre los 1 I a 2 I años.

Estos propósitos están expresados, en el objetivo número cuatro, que manifiesta "La integración de la escuela en la comunidad [...]", en el séptimo en donde se dice "Promover la generación de un ámbito específico para la apertura de la escuela hacia la comunidad que incorpore población juvenil socialmente desfavorecida", en el décimo que destaca "Ampliar y fortalecer los vínculos establecidos desde la Escuela con los distintos sectores de la comunidad..." y por último en el décimo tercero "Optimizar el uso de los recursos e infraestructura disponibles en la Escuela, el barrio y el distrito" (DGCYE, 2006b). Estos planteos se centran en la utilización de la escuela de una forma distinta a la habitual, como un lugar de encuentro, abierto para toda la población, para la realización de distinto tipo de actividades, función que en otros momentos de la historia estaban focalizadas también en los clubes, sindicatos etc. 
Considerando todos los objetivos del programa, podemos ver la existencia de un problema central que es la falta de propuestas y espacios para ocupar el tiempo libre de los jóvenes, lo cual se asocia con la permanencia en la calle, con las consecuentes posibilidades de riesgo tanto para ellos, como para la comunidad.

Esta falta de espacios se encuentra ligada en cierta medida al proceso de privatización que sufrió el país a lo largo de la década de 1990, con el gerenciamiento de los clubes y a la utilización de espacios públicos para la construcción de diversas iniciativas privadas como son las canchas de fútbol o de diversiones, así también como consecuencia del crecimiento de la ciudad, que fue ocasionando una paulatina disminución de los espacios en donde los jóvenes y la población en general pueda acceder a diversos tipos de actividades, de forma gratuita o de bajo costo.

En suma, la apertura de la escuela los fines de semana, con actividades no curriculares, es vista, desde la fundamentaciones de los proyectos como una forma de soportar el presente con talleres de su agrado.

Continuando con la lógica de razonamiento que nos propone los documentos del programa, para lograr una efectiva participación, las temáticas propuestas deben ser de interés para los jóvenes pues traerá como consecuencia su asistencia a la escuela, cumpliéndose la ponencia central del programa.

En el manual de procedimientos del programa del año 2006 encontramos que las categorías temáticas de las actividades que se pueden desarrollar son las siguientes:

- Arte y cultura: teatro, música etc.

- Actividades de contención social: informativas, SIDA, droga, interés general etc.

- Comunicación.

- Participación en la comunidad: voluntariado social, actividades solidarias etc.

- Actividades de capacitación en oficios múltiples o en oficios no convencionales.

- Prácticas corporales.

En relación a las temáticas, no se ha observado ninguna fundamentación sobre su incorporación, y considerando las propuestas de los proyectos, inferimos que el centro no es si el taller es de teatro o deporte, sino que éste sea atractivo para los jóvenes, lo cual va a favorecer la permanencia y la asistencia al edificio escolar los fines de semana. 


\section{LAS PRÁCTICAS CORPORALES EN PROGRAMAS SOCIALES EN EL BRASIL}

Otras experiencias similares al Programa "Patios Abiertos en las Escuelas", permiten ampliar el espectro y dimensionar la magnitud de la problemática en discusión, sobre la inclusión de las prácticas corporales en estas políticas socio-educativas. Con dicha intención se presentarán una serie de experiencias para contrastar los fundamentos de estas prácticas. La búsqueda y selección de las experiencias se realizó con el criterio que respondieran a las siguientes propuestas: que se desarrollen prácticas corporales, que se ejecuten en establecimientos educativos, que tengan como destinatarios a la población comprendida entre los I I a 24 años, y que las mismas sean consideradas iniciativas de inclusión educativa. Esto posibilitó constatar que existen experiencias similares en Latinoamérica, mostrando una misma metodología de funcionamiento, ante la misma población, expresando en los distintos casos como problemática: el tiempo libre de los jóvenes fuera del espacio escolar, asignándole en este contexto a las prácticas corporales una función que es la de atraer a los jóvenes hacia instituciones educativas ${ }^{7}$.

Las experiencias a presentar se organizarán según la institución encargada de su ejecución. En un primer momento se relevarán tres programas desarrollados por la Unesco Brasil; luego el Programa Estadual de Esporte Escolar, Santa Catarina y por último el Programa Segundo Tempo.

\section{PROGRAMAS DE UNESCO BRASIL, "ESCOLAS DE PAZ"}

Los primeros programas que se encontraron fueron aquellos encuadrados dentro de la experiencia "Escolas de Paz", antecedente del programa en análisis. La metodología de funcionamiento de las tres iniciativas consiste en la apertura de los edificios escolares los fines de semana, ofreciendo diversos talleres, entre ellos, con mayor concurrencia, en los que se dictan prácticas corporales.

A continuación tomaremos en cuenta la función asignada a las prácticas corporales en estas tres iniciativas. Según el orden cronológico de su aparición, se encuentra: el "Programa Abrindo Espaços: Educação e Cultura para a Paz" en ejecución en el estado de Bahía, en el cual la incorporación de las prácticas corporales está asociada a las posibilidades que presentan estas actividades en cuanto a realizar

7. Se han encontrado otras experiencias similares además de las presentas a continuación en el texto, entre las que se pueden destacar: en Argentina (Plan Escuelas Abiertas: en la Provincia de Salta, Programa Club de Jóvenes en la Ciudad Autónoma de Buenos Aires, los Centro de Actividades Juveniles que se encuentran en todas las provincias del país) y en el Brasil (Programa Escola Comunidade en el estado de Piaú, Abrindo Espaços na Escola Viva, Comunidade Ativa en el estado de Minas Gerais y Escola Aberta para a Cidadania, en el estado de Rio Grande do Sul). 
trabajos en equipos, estimulando la cooperación, como así también la valoración e incorporación del capital cultural de los jóvenes dentro de la institución escolar, como se destaca en la evaluación realizada por Miriam Abramovay (2003) ${ }^{8}$. En el programa, rescatamos que en el 81,7\% de los talleres se desarrollan Prácticas Corporales, principalmente Capoeira, Danza, Futsal, Voley, Fútbol de campo y por último Básquet. Éstos también son los que tienen mayor número de participantes, principalmente en los talleres de Capoeira.

El "Projeto Escola Aberta" del estado de Pernambuco asocia la falta de espacios para la realización de actividades deportivas con los hechos de violencia, a su vez se encuentra nuevamente la valoración del capital cultural de los jóvenes para incorporarlos dentro de las escuelas.

En tercer lugar, "Escolas de Paz" del estado de Río de Janeiro, en cuyos documentos no se ha encontrado fundamentación en torno a la inclusión de las prácticas corporales. En la evaluación realizada por Waiselfisz (2003), se observa la necesidad de apertura de espacios en los que se desarrollen actividades culturales como medio para la reducción de la violencia, como fue planteado al inicio de la presentación de este programa.

\section{PROGRAMA ESTADUAL DE ESPORTE ESCOLAR, SANTA CATARINA}

El Programa Estadual Esporte Escolar, fue creado en el año 200 I, comenzando a funcionar en 75 escuelas en el segundo semestre de dicho año, ampliando a 293 establecimientos educativos en el año 2002, continuando su funcionamiento hasta fines del año 2003 (OLIVEIRA, 2006). Esta iniciativa considera que las prácticas corporales contribuyen a la inclusión social, al desenvolvimiento del individuo y al ejercicio de la ciudadanía.

Esta iniciativa está orientada a niños y jóvenes mayores de 10 años, pudiendo participar menores a esa edad en las actividades de Danza y Recreación. A todos los participantes se les exigía encontrarse matriculados en el "Ensino Fundamental" en escuelas públicas y estatales. Es propio destacar que dado el caso que los alumnos bajen su desempeño escolar, no pueden participar del programa, pero sí retornarlo en el momento de elevar sus notas.

Lo que se expuso hasta el momento fue la información del programa disponible en las "Diretrizes para implantação/implementação do Programa Estadual

8. "[...] as atividades esportivas (capoeira, karatê, futebol), experimentando regras que estimulem a cooperação e o trabalho em equipe" (ABRAMOVAY, 2003, p. 50). 
Esporte Escolar" de la "Secretaria de Estado da Educação e Inovação" del Estado de Santa Catarina.

En el trabajo "Políticas Publicas de Esporte Escolar e Educação Física Escolar: entre a inclusão social e a busca por talentos esportivos, tendo como pano do fundo o Programa Estadual Esporte Escolar de Santa Catarina" realizado por Luis Carlos de Jesus Gaspar; Santiago Pich, Alexandre Fernandez Vaz, nos muestra la dicotomía de los documentos y las afirmaciones de los profesores a cargo de las actividades, mostrando como esta experiencia con fines socio-educativos en los documentos, se transforma en la formación de equipos para competir en torneos municipales, lo cual como destacan los autores "[...] generando assim uma expectativa en seus participantes quanto a possiveis conquistas de carater pessoal [...]" (GASPAR; PICH; VAZ, 2004, p. 114).

\section{PROGRAMA "SEGUNDO TEMPO"}

El programa "Segundo Tempo" es una iniciativa del Ministerio de Deportes, en relación con el Ministerio de Educación, ambos del Brasil, que se encuentra bajo la órbita de la "Secretaria Nacional de Esporte Escolar" del primero de los ministerios. Consiste en el dictado de clases de distintos deportes en los establecimientos escolares en el contra turno, teniendo como objetivos "[ . . . ] a democratizaro acesso à prática esportiva, por meio de atividades esportivas e de lazer [...]", buscando la inclusión social, ocupar el tiempo de ocio de los niños y los adolescentes en situación de riego social.

Éste fue creado en el año 2003 y comenzó su implementación en el año 2004, funcionando hasta la actualidad y siendo desde el principio la política central del "Ministerio do Esporte"

\section{CONSIDERACIONES FINALES}

Este trabajo abordó y presentó el sentido y la función asignada a las prácticas corporales, en distintas experiencias de políticas socio-educativas, orientadas a los jóvenes, profundizando el análisis en el Programa "Patios Abiertos en las Escuelas".

En las fundamentaciones del programa "Patios Abiertos en las Escuelas" se observa como las prácticas corporales funcionan como atractivo para la permanencia de los jóvenes en las escuelas más allá del tiempo curricular, con el fin de ocupar el tiempo libre en un ambiente institucionalizado, reduciéndose de esa forma el riesgo potencial. 
Este tipo de experiencias son aprovechadas, en la práctica, para generar lugares de aprendizaje de las distintas prácticas corporales y no como un mero propósito de controlar una población que se percibe como riesgosa.

Encontramos en las distintas experiencias lineamientos comunes. En las seis experiencias presentadas, las fundamentaciones se centran en la ocupación del tiempo libre de los jóvenes. La existencia de éste aparece consecuentemente asociado con la permanencia en las calles, con las posibles actividades peligrosas que se pueden realizar en este medio, con la falta de ofertas gratuitas para ocupar dicho bache temporal y principalmente con la inclinación personal hacia las prácticas corporales. Estos factores que componen el discurso que legitima a las prácticas corporales dentro de estas iniciativas las presentan como un señuelo para atraerlos a las escuelas.

Entre estos argumentos, aparece en las fundamentaciones relegada la cuestión del aprendizaje de una práctica corporal, el acceso a la cultura que permite la apertura de las escuelas los fines de semana, que en muchos casos se transforma en el único canal. Al buscar la procedencia de este discurso generalizado sobre las prácticas corporales dentro de experiencias de políticas socio-educativas se halló una correlación directa con las proclamas de la UNESCO y las Naciones Unidas en el marco del anuncio del año 2005 como "Año Internacional del Deporte y la Educación Física".

En los argumentos de estos organismos internacionales, se observa una preponderancia de las prácticas deportivas por sobre otras prácticas corporales, asignándoles distintos propósitos, como se puede ver en la fundamentación de la ONU. Se expresa en el apartado "El Deporte y los Objetivos de Desarrollo del Milenio" el siguiente objetivo: "Lograr la educación primaria universal" en donde se destaca que: "Las actividades deportivas y la Educación Física hacen que la escuela sea más atractiva y que se incremente la asistencia de los estudiantes". Se observa en la comparación de los documentos de los distintos programas y organismos similares argumentos sobre la inclusión de las prácticas corporales, que en muchos casos, se encuentran determinadas por los organismos internacionales encargados del financiamiento, evaluación o que realizan el asesoramiento, en nuestro caso en estudio la UNESCO, por dicha razón se plantea la necesidad de realizar estudios comparados y sistemáticos entre las distintas experiencia a nivel global, para poder comprender la compleja trama que atraviesa a las políticas educativas que incorporan a las prácticas de la Educación Física, atendiendo al mismo tiempo a los procesos de apropiación y resignificación que se suscitan en cada caso particular por los distintos actores que participan de las mismas. Para tener una comprensión amplia de éstas se debe indagar profundamente en las representaciones de las prácticas corporales 
de los profesores que se desempeñan en los distintos niveles de gestión, los que dictan las actividades y los jóvenes participantes, manteniendo comparaciones con diversas experiencias.

\section{A educação física em políticas socioeducativas destinadas aos jovens}

RESUMO: Este trabalho tem como propósito abordar a problemática das práticas corporais imersas nas políticas socioeducativas da Dirección General de Cultura y Educación (DGCyE) da Província de Buenos Aires, Argentina, em particular o sentido e a função destinadas a elas no programa "Patios Abiertos en las Escuelas", projeto destinado aos jovens. Mantendo em dita abordagem comparações com outras pesquisas e com documentos de iniciativas similares que se desenvolvem no Brasil: "Abrindo Espaços", executado pela Unesco, o "Programa Estadual de Esporte Escolar" do estado de Santa Catarina e o programa "Segundo Tempo", iniciativa do Ministério do Esporte, indaga-se sobre as linhas de fundamentação comuns entre eles.

PALAVRAS-CHAVE: Educação física; políticas socioeducativas; jovens; práticas corporais.

\section{Physical education in educational associate policies destined to young people}

ABSTRACT: This work aims to reach the problem of embodied practices embedded in social and educational policies of the General Directorate of Culture and Education of the Province of Buenos Aires Argentina, and in particular the sense and the assigned function to them in the "Open places in Schools" program for young people.

Keeping this approach in comparison with other research and documents that are developing similar initiatives in Brazil: "Opening Spaces" executed by Unesco and the "Statewide program of School Sports" in the state of Santa Catarina and the "Second Time" program, is an initiative of the Sports Ministry, asking about the lines of support assigned.

KEY WORDS: Physical education; educational associate polities; young; body practices.

\section{REFERENCIAS}

ABRAMOVAY, M. Avaliação do Programa Abrindo Espaços na Bahia. Brasília: Unesco, Universidade Católica de Brasília, Unirio, 2003.

BOURDIEU, P. Sociología y cultura. México, Grijalbo, 1990.

BUENOS AIRES. Resolución n. 4235/2003 del 8 de octubre de 2003. Protocolo de cooperación. Dirección General de cultura y Educación y la Organización de las Naciones para la Educación, la Ciencia y la Cultura - Unesco. DGCyE, 2003.

- Resolución n. 100/2004 del 30 de enero de 2004. Crear la Dirección provincial de política socio - educativa. DGCyE, 2004. 
- Resolución n. 2273/2004 del 8 de julio de 2004. Creación del Programa Patios Abiertos en las Escuelas. DGCyE, 2004.

. Resolución n. 3328/2004 del 22 de septiembre de 2004. Incorporación a partir del I de agosto de 2004 los proyectos presentados en Anexo I. DGCyE, 2004.

. Resolución n. 72/2006. Decretar del interés Provincial al Programa Patios Abiertos en las Escuelas. DGCyE, 2006 a.

. Manual de Procedimientos 2006. Buenos Aires: DGCyE, 2006b.

CASTEL, R. La metamorfosis de la cuestión social. México: Paidos, 1997.

CHAVES, M. Juventud Negada y Negativizada: Representaciones y formaciones discursivas vigentes en la Argentina contemporánea. Revista Ultima Década. Viña del Mar Chile, n. 23, p. 9-23, CIDPA, 2005.

GASPAR, L. C. J.; PICH, S.; VAZ, A. F. Política pública de esporte escolar e Educação Física escolar: entre a inclusão social e a busca por talentos esportivos, tendo como pano de fundo o Programa Estadual de Escolar de Santa Catarina. Florianópolis. Motrivivência, v. XVI, n. 23, p. |03-| |6, 2004.

GIL, A. C. Como elaborar projetos de pesquisa. 3. ed. São Paulo: Atlas, 199I.

GOMES, C. Dos valores proclamados aos valores vividos: traduzindo em atos, principios das Naçoes Unidas e da Unesco para projetos escolares e politicas educacionais. Unesco, 2001.

HOMRICH de CECCO, L., Prioridades da Política de Esporte Escolar no Brasil. In KUNZ, E.; HILDEBRANDT-STRAMANN, R. (Org.). Intercâmbios científicos Internacionais em Educação Física e Esporte. Rio Grande do Sul: Ed. Unijui, 2004.

MARLOVAJOVCHELOVITCH, N.; GARCIA CASTRO, M.; ABRAMOVAY, M. Abrindo espaços: educação e cultura para a paz. 3.ed. Brasília: Unesco, 2004.

MINAYO, Maria Cecília de S. (Org.). Pesquisa Social. Teoria, método e criatividade. Rio de Janeiro: Petrópolis, 1994.

OLIVEIRA, P. A esportivização das políticas públicas?: análise de programas para a infância e a juventude no Estado de Santa Catarina. Dissertação (Mestrado) - Programa de Pós-Graduação em Educação Física, UFSC, Florianópolis, 2006.

REGUILLO CRUZ, R. Crónica roja: espectáculo y negocio. Jóvenes: la construcción del enemigo. Chasqui Revista Latinoamericana de Comunicación. n. 60 diciembre, 1997. Disponible en: <http://www.comunica.org/chasqui/reguillo.htm>. Acceso en: 8 nov. 2007.

. Emergencias de culturas juveniles. Estrategias del desencanto. I. ed. Bogota: Grupo Editorial Norma, 2000. 
WAISELFISZ, J.; Maciel, M. Revertendo violências, semeando futuros: avaliação de impacto do Programa Abrindo Espaços no Rio de Janeiro e em Pernambuco. Brasília: Unesco, 2003.

Recebido: 7 jul. 2009 Aprovado: 10 maio 2010

Endereço para correspondência:

Alejo Levoratti Calle 387 n. 3805

Quilmes Oeste, Buenos Aires, Argentina

CEP: 1879 